\title{
Epileptiform Activity in the Guinea-pig Neocortical Slice Spreads Preferentially along Supragranular Layers- Recordings with Voltage-sensitive Dyes
}

\author{
Birgit Albowitz ${ }^{1,2}$ and Ulrich Kuhnt ${ }^{1}$ \\ 1Department of Neurobiology, Max-Planck-Institute for Biophysical Chemistry, PO Box 2841, 37018 Göttingen, Germany \\ 2Institute of Experimental Epilepsy Research, University of Münster, Münster, Germany
}

Key words: epileptiform activity, pathways of spread, horizontal connections, neocortex, voltage-sensitive dyes, guinea-pig

\begin{abstract}
The spread of epileptiform activity was monitored in guinea-pig neocortical slices by the use of a voltagesensitive dye (RH795) and a fast optical recording technique. Epileptiform activity induced by bicuculline methiodide $(10-20 \mu \mathrm{M})$ and single-pulse stimulation spread from the stimulation site in layer I or in the white matter across most of the slice. Different lesions were made in the slice in order to specify the neuronal connections used for spread in the horizontal direction. In the slice, intracortical connections are necessary for the spread of epileptiform activity, as shown by vertical cuts through all cortical layers but sparing the white matter. Horizontal connections were interrupted by cuts parallel to the axis of pyramidal neurons through either supragranular or infragranular layers. Vertical connections were interrupted by cuts perpendicular to the axis of pyramidal neurons separating supragranular and infragranular layers. Spread of epileptiform activity in the horizontal direction was not hindered by horizontal cuts. Vertical cuts through infragranular layers also did not hinder the spread of epileptiform activity. In contrast, vertical cuts through supragranular layers either abolished completely (nine slices) or delayed significantly (ten slices) the spread of epileptiform activity. The mean delay at the supragranular lesion was $44 \mathrm{~ms}$ in layer III and $30 \mathrm{~ms}$ in layer V; at the infragranular lesion the mean delay was $2 \mathrm{~ms}$ in layer III and $6 \mathrm{~ms}$ in layer V. Also, with horizontal cuts, in three out of five slices the velocity of spread was significantly lower in infragranular as compared to supragranular layers. It is concluded that both supra- and infragranular layers if isolated possess the ability to initiate and propagate epileptiform activity independently. However, in the intact slice the influence of the supragranular networks on initiation and propagation of epileptiform activity appears to dominate.
\end{abstract}

\section{Introduction}

Epileptiform discharges are characterized by paroxysmal depolarization shifts at the single-cell level (Matsumoto and Ajmone-Marsan, 1964), by synchronicity of such discharges among neurons (e.g. Traub and Wong, 1982) and by spread across wide areas of the brain (e.g. Goldensohn and Salazar, 1986; London et al., 1989). In vivo, the spread of epileptiform activity uses major anatomical pathways including subcortical-cortical routes (Gutnick and Prince, 1975; Schwartzkroin et al., 1975; Kusske, 1976). Spread also occurs in the in vitro slice preparation, however, at a lower velocity (Chervin et al., 1988; Chagnac-Amitai and Connors, 1989a; Albowitz et al., 1990; Wong and Prince, 1990; Albowitz and Kuhnt, 1993b; Wadman and Gutnick, 1993). The purpose of this investigation was to locate the neuronal connections that are most favourably used for the propagation of epileptiform activity in the neocortical slice.

In the neocortical slice, the spread of epileptiform activity has been monitored by extracellular field potential recordings (Chervin et al., 1988; Chagnac-Amitai and Connors, 1989a, b; Wadman and Gutnick, 1993) as well as by optical recording with voltage-sensitive dyes
(Albowitz et al., 1990; Albowitz and Kuhnt, 1993b). In these studies the neuronal structures and connections preferentially used for the spread of epileptiform activity in the horizontal direction could not be determined.

Excitatory synaptic connections are likely to be involved in the spread of activity (Gutnick et al., 1982; Traub and Wong, 1982). Spread might be mediated by excitatory fibres leaving and re-entering the grey matter or by several intracortical routes: vertical connections, e.g. axon collaterals connecting layer III and layer V pyramidal cells (Gilbert and Kelly, 1975; Tigges and Tigges, 1982) or connections made by layer IV spiny stellate cells (Gilbert and Wiesel, 1979; Martin and Whitteridge, 1984) might propagate epileptiform activity in the horizontal direction by a 'reverberating' mechanism; horizontal connections, e.g. short- and long-range axon collaterals of pyramidal cells (Fisken et al., 1975; Gilbert and Wiesel, 1979) found in all cortical layers (Creutzfeldt et al., 1977) would be well suited for horizontal propagation. If horizontal connections play a role, the spread of epileptiform activity might use all or predominantly 
connections in either supragranular or infragranular layers. In this context, excitatory synaptic connections between intrinsically bursting cells of layers IV and V are of particular interest. It was proposed that these cells cause synchronization and spread of epileptiform activity (Chagnac-Amitai and Connors, 1989b).

In order to determine the neuronal structures and connections that are necessary or most favourable for the spread of activity, slices were sectioned at specific sites and the influence of such lesions on the spread of epileptiform activity was determined. We used a voltagesensitive dye and a fast optical recording technique to monitor epileptiform activity non-invasively, at multiple sites, and with sufficient spatial and temporal resolution. Some of these results have been published in abstract form (Albowitz and Kuhnt, 1993d).

\section{Materials and methods}

\section{Preparation}

Adult guinea-pigs $(400-600 \mathrm{~g})$ were killed by a blow to the neck and then decapitated. After removing the skull, the brain was placed in ice-cold oxygenated medium. A block of tissue was prepared from the caudal half of the cerebral cortex. Ventrally, the block was trimmed below the white matter. Coronal sections of $350 \mu \mathrm{m}$ thickness were cut in ice-cold oxygenated medium on a rotorslicer (Dosaka, Kyoto, Japan). Slices were kept in oxygenated medium in a storage chamber at $28^{\circ} \mathrm{C}$. They were stained in a separate compartment of the storage chamber filled with voltage-sensitive dye solution, and gassed with a mixture of $\mathrm{O}_{2} / \mathrm{CO}_{2}(95 \% / 5 \%)$. The voltage-sensitive fluorescence dye RH795 (Grinvald et al, 1986) (Molecular Probes, Eugene, OR) was used, dissolved in standard medium $(50-100 \mu \mathrm{g} / \mathrm{ml})$ with $0.5 \%$ dimethyl sulphoxide (DMSO). In control experiments, evoked field potentials recorded during application of DMSO $(0.5 \%)$ via the standard medium were not affected. The slices were stained for at least $1 \mathrm{~h}$, washed briefly in the storage chamber, and then transferred to the recording chamber, where they were perfused for another hour before optical recording was started. In some preliminary experiments, slices were stained in the recording chamber while continuously recording evoked field potentials. No changes in the size or shape of field potentials occurred during or after the staining procedure.

\section{Recording chamber, electrical recording and stimulation}

Recordings were made in a superfusion chamber at a temperature of $32 \pm 0.5^{\circ} \mathrm{C}$. The chamber was perfused at a rate of $5 \mathrm{ml} / \mathrm{h}$ (chamber volume $\sim 0.5 \mathrm{ml}$ ) with a standard medium containing $124 \mathrm{mM} \mathrm{NaCl}$, $5 \mathrm{mM} \mathrm{KCl}, 1.25 \mathrm{mM} \mathrm{NaH} \mathrm{PO}_{4}, 2 \mathrm{mM} \mathrm{MgSO}, 2 \mathrm{mM} \mathrm{CaCl}_{2}, 26$ $\mathrm{mM} \mathrm{NaHCO} 3$ and $10 \mathrm{mM}$ glucose. The medium was gassed with $\mathrm{O}_{2} / \mathrm{CO}_{2}$ giving a $\mathrm{pH}$ of 7.4. The humidified $\mathrm{O}_{2} / \mathrm{CO}_{2}$ mixture was continuously blown over the surface of the medium in the recording chamber. Extracellular field potentials were recorded with glass pipettes filled with $3 \mathrm{M} \mathrm{NaCl}(5-10 \mathrm{M} \Omega)$. Two stimulation electrodes (tungsten in glass, $50-200 \mathrm{k} \Omega$, tip diameter $<30 \mu \mathrm{m}$ ) were inserted, one at the border between grey and white matter (white matter), the other in layer I. Both sites were stimulated separately. Single pulses (width $30 \mu$ s) with an intensity ranging from 7 to $40 \mathrm{~V}$ were used at a rate of $0.05 \mathrm{~Hz}$.

\section{Experimental protocol}

Following control recordings, bicuculline methiodide was added to the bath medium $(10-20 \mu \mathrm{M})$. Epileptiform potentials were then elicited by single-pulse stimulation with an intensity sufficient to evoke reliably epileptiform events at a constant latency. Spread of epileptiform activity in intact slices $(n=15$; Albowitz and Kuhnt, 1993b) was compared with the spread in slices with specific cuts $(n=28)$. Cuts were either parallel or perpendicular to the axis of pyramidal neurons. They were made following the staining procedure with a scalpel prepared from a razor blade.

\section{Optical recordings}

A detailed description of the optical recording method is provided in a previous publication (Albowitz et al., 1993a). Briefly, an inverted microscope (IM35; Zeiss, Oberkochen, Germany) was mounted on an $x-y$ table below a rigid stand holding the recording chamber and micromanipulators. The activity-dependent fluorescent light was projected onto a $10 \times 10$ photodiode array (Centronic; MD100-5, Croydon, UK), which was placed in the real image plane of the microscope. According to the objective used $(\times 16$ Zeiss Neofluar) a spatial resolution of $94 \mu \mathrm{m}$ was achieved. Therefore, activity was detected simultaneously from a total area of $(940 \mu \mathrm{m})^{2}$. In order to monitor the spread of epileptiform activity across the entire slice, the microscope with the attached photodiode array was moved below the stationary slice by use of the $x-y$ table. The precision of the movement provided by the $x-y$ table is $5 \mu \mathrm{m}$. The photodiode array was moved such that one row of photodiodes overlapped at two adjacent positions; in this way one row of photodiodes served for control measurements. An additional control for the stability of epileptiform events was the continuous recording of field potentials from a stationary site in layer III. Only those experiments for which field potential recordings were stable from stimulus to stimulus for one experimental condition were considered for further analysis. The signals from each photodiode were separately current-to-voltage converted, amplified, and processed by a computer system. The system permitted a time resolution of 0.4 ms. Single sweeps were recorded. Following data acquisition, a photograph of each position of the slice was taken with a scheme of the photodiode array superimposed.

\section{Data analysis}

Signals were weakly smoothed (each data point was calculated as the average of its own value weighted $6 / 8$ and the values of each neighbouring point weighted 1/8 each). Dye bleaching, staining and illumination irregularities were corrected off-line by expressing light intensities as relative fluorescence changes $(\mathrm{d} F / F)$, where $F$ is the fluorescence light intensity of the stained slice during illumination without evoked neuronal activity, $\mathrm{d} F$ is the fluorescence change during evoked neuronal activity. A decrease in fluorescence (plotted upwards in all records shown) corresponds to membrane depolarization; an increase in fluorescence corresponds to membrane hyperpolarization.

For each record, a baseline was calculated as the average amplitude of the 250 data points $(100 \mathrm{~ms})$ preceding the stimulus. The first point after the stimulus that was followed by at least 100 data points that were above baseline was defined as onset of the epileptiform potential (e.g. Fig. 2D). For each record, a window of $100 \mathrm{~ms}$ was set from this onset point. From these window settings, onset latencies, mean window amplitudes and occasionally maximal amplitudes were determined. The onset latency was the latency from stimulation to onset of the potential, the mean window amplitude was the average of the amplitude values from all data points within the window. These values were used to construct three-dimensional graphs with 'distanceweighted least-squares' smoothing (SYSTAT for Windows; SYSTAT, 
Evanston, IL). In addition, individual values were plotted along specific cortical layers in order to provide a more quantitative analysis. All values are from single responses.

\section{Histological procedures}

The position of the photodiode array with respect to the slice and to the stimulation electrodes was determined photographically in situ. Following the recording procedure, the slices were placed in fixative composed of $1 \%$ formaldehyde, $1.5 \%$ glutaraldehyde, $4 \%$ sucrose and $0.1 \mathrm{M}$ phosphate buffer in distilled water. Frozen sections of $52 \mu \mathrm{m}$ thickness were cut parallel to the surface of the slices and stained with cresyl violet. Shrinkage of slices due to fixation was compensated for by correlating the electrode position on the photographs taken in situ and the lesions induced by the electrodes in the histological sections. Only those experiments in which no patches of damaged cells (swollen or pycnotic) except near the lateral cutting line, near the cuts, and around the stimulation electrodes could be detected were considered for further analysis. Cortical areas (Wree et al., 1981) and layers were identified light microscopically and correlated with the positions of the photodiode matrix. No attempt was made to separate layers II and III (see also Braitenberg and Schüz, 1991). The extents of the induced lesions and the affected cortical layers were determined. Cuts were inspected for completeness in depth and for the amount of tissue damage on their borders. Typically, $<30 \mu \mathrm{m}$ of tissue around the cut was damaged (as determined light microscopically) due to the sectioning procedure (Fig. 1).

\section{Results}

In the stained neocortical slice, single-pulse stimulation of either white matter or layer I evoked decreases in fluorescence light intensity (i.e. summated increases of membrane depolarization) in a restricted area around the stimulation electrode (Albowitz and Kuhnt, 1993a) (Fig. 2A, E). As reported previously (Albowitz and Kuhnt, 1993b), following the addition of bicuculline, stimulation evoked single epileptiform events which lasted up to $1 \mathrm{~s}$ and spread across almost

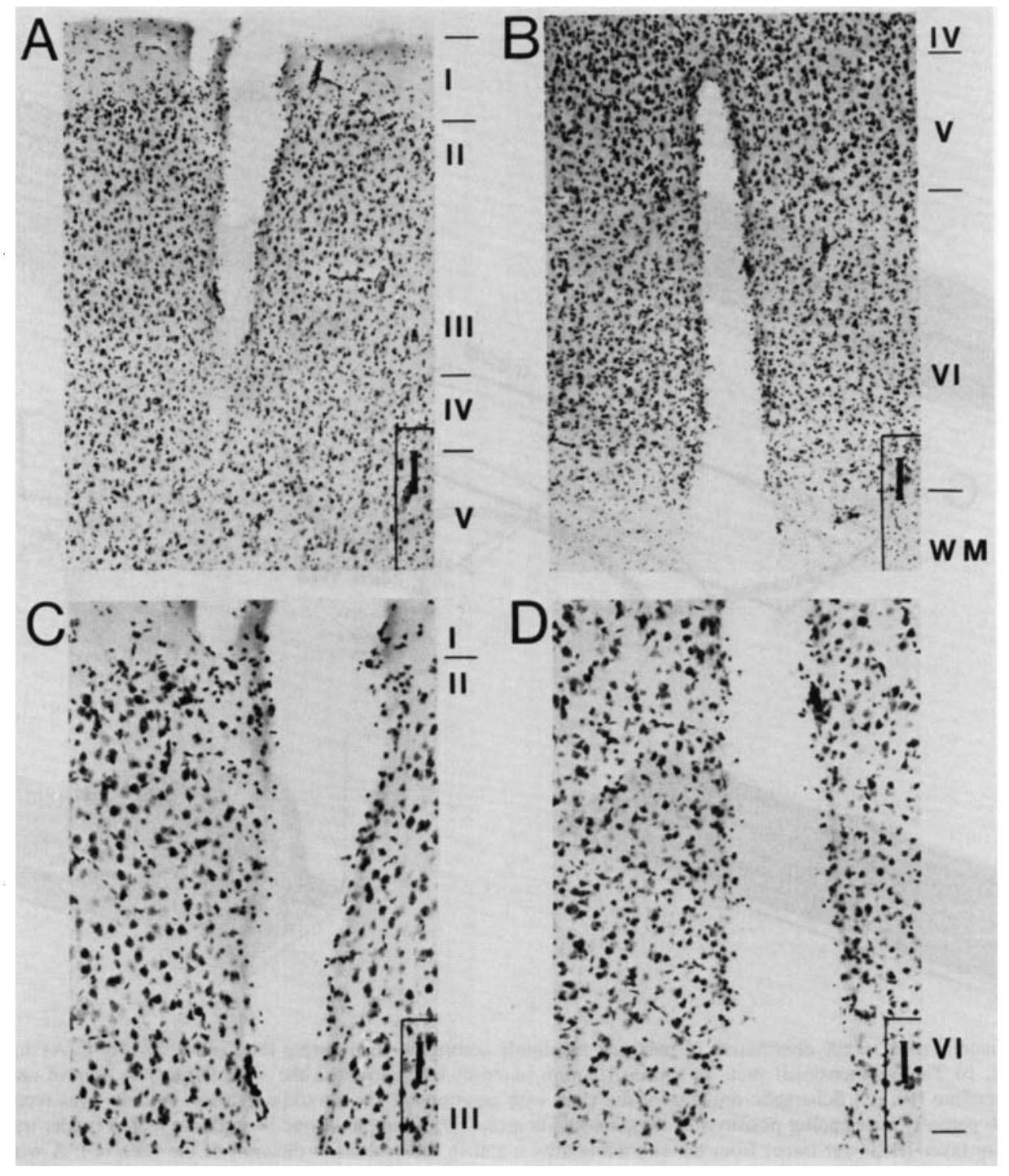

FIG. 1. Photomicrographs from a histological section (cresyl violet staining) depicting a vertical lesion through supragranular (A) and infragranular (B) layers. (C, D) Enlarged sections from A and B. Calibration bars: $100 \mu \mathrm{m}$ (A, B) and $50 \mu \mathrm{m}$ (C, D). 
the entire slice (Fig. 2B, F). Electrophysiologically, these events resemble interictal potentials in vivo. The distribution of amplitudes and onset latencies of control and epileptiform potentials across the slice is illustrated in Figure 2.

The distribution of onset latencies (Fig. 2F) did not permit us to determine whether neuronal connections in specific layers are used for the spread of epileptiform activity in the horizontal direction, such that these layers would be activated prior to other structures. Onset latencies of epileptiform activity in supra- and infragranular layers differed only minimally and the differences were not statistically significant (Fig. 2F). Therefore, another approach was necessary to identify neuronal connections essential for the spread of epileptiform activity.

\section{Vertical lesions across the grey matter}

In two slices, vertical cuts were made through the entire grey matter, sparing the white matter. In these slices, epileptiform activity did not spread past this lesion (Fig. 3). Epileptiform activity could be initiated by stimulation of the white matter or layer I both medially and laterally from the lesion. Activity spread from the respective stimulation site up to $100 \mu \mathrm{m}$ from the lesion; responses declined thereafter. Thus, in the slice, fibres leaving the grey matter and re-entering it at more distant locations are not sufficient for the spread of epileptiform activity.

\section{Horizontal lesions}

Infragranular and supragranular layers were separated in seven slices for a length of $\sim 2 \mathrm{~mm}$ by a horizontal cut, which started at the lateral edge of the slice. All sections were made along layer IV. Thus, in the course of the section, a variable part of layer IV was either found in the supragranular or in the infragranular part of the slice. The amount of layer IV present in each case did not influence the results. Stimulation electrodes were placed in layer I and the white matter either within the lesioned part of the slice (all of seven slices; Fig. 4), or at a distance of at least $3 \mathrm{~mm}$ from the lesion, usually at the medial edge of the slice (five of seven slices; Fig. 5).

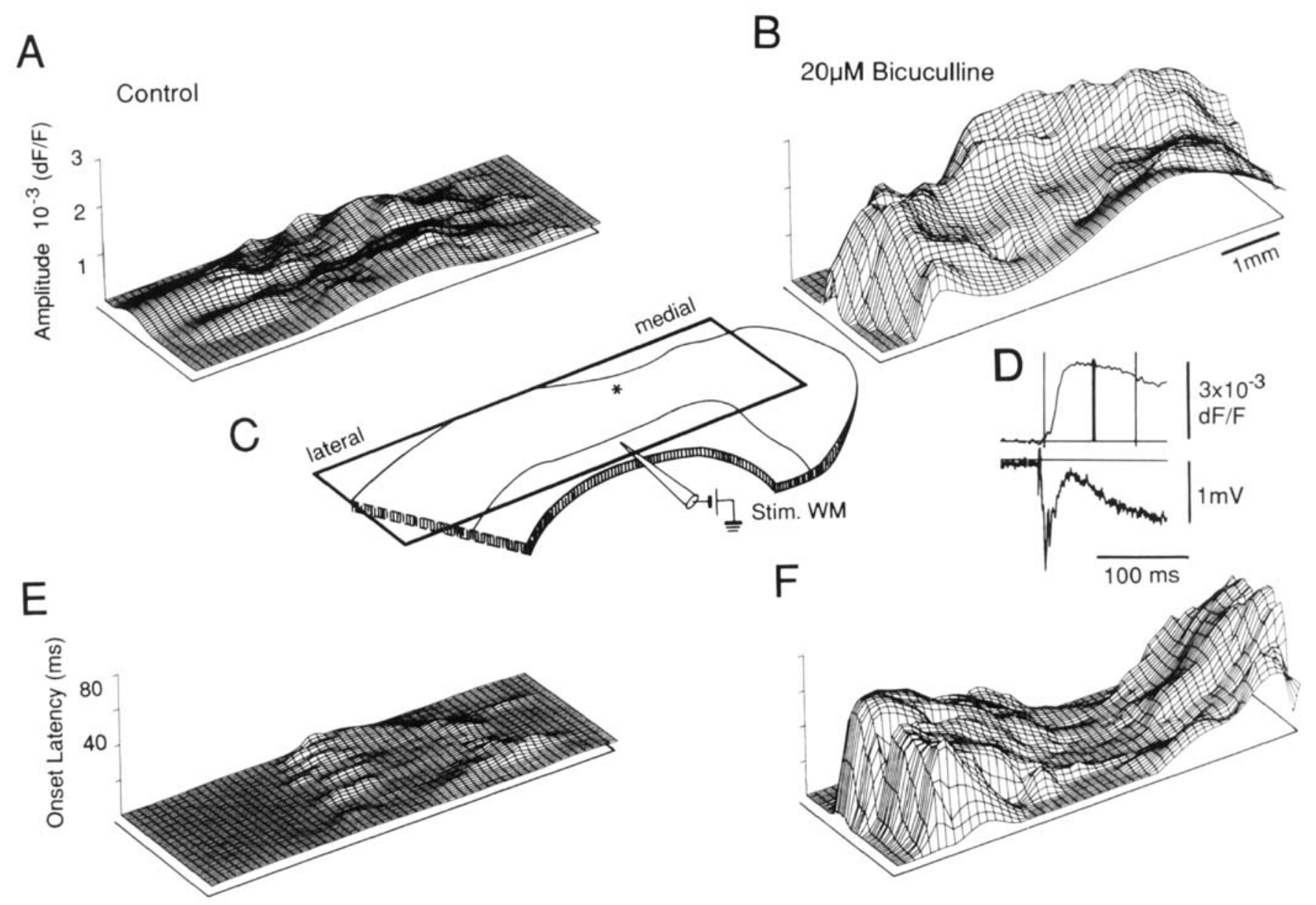

FIG. 2. (A, B) Three-dimensional plots of the distribution of maximal amplitude across the slice during the control condition (A) and during the application of $20 \mu \mathrm{M}$ bicuculline (B). (E, F) Three-dimensional plots of the distribution of onset latency across the slice during the control condition (E) and during the application of $20 \mu \mathrm{M}$ bicuculline (F). (C) Schematic drawing of the slice with positions of the photodiode array. Activity was recorded following stimulation of the white matter from 16 partially overlapping positions of the photodiode array. (D) Example of one photodiode record (upper trace) and the simultaneously recorded field potential from layer III (lower trace) from the site marked by a star in the schematic diagram of the slice (C). A window of $100 \mathrm{~ms}$ was set at the onset of the optically recorded epileptiform potential. From this window, onset latency and maximal amplitude (heavy vertical bar) were determined. These measurements were made for all 1600 photodiode records. The values were then used to construct the three-dimensional plots shown. Note that the $z$-axis in $\mathrm{A}, \mathrm{B}, \mathrm{E}$ and $\mathrm{F}$ and the $y$-axis in $\mathrm{D}$ all indicate the amplitude of the response. However, the $x$ - and $y$-axes in A, B, E and F represent the spatial surface of the slice whereas the $x$-axis in D represents time. 
In the first case, epileptiform activity had to be initiated in the lesioned part of the slice. For stimulation of the white matter, epileptiform activity had to be generated in the infragranular part only; for stimulation of layer I only supragranular layers were available for the generation of epileptiform activity. Independent from the stimulation site, epileptiform activity spread to all areas of the slices except for a small band of tissue close to the cut (Fig. 4A, D). Thus, epileptiform activity can be initiated separately in supra- or in infragranular structures. Propagation started from the point of stimulation in the lesioned part and epileptiform activity invaded both supra- and infragranular layers in the lesioned and the intact part of the slice.

If stimulation electrodes were set distant from the lesion in the intact part of the slice, epileptiform activity was initiated there and spread into the lesioned part of the slice (Fig. 5). Again, all areas of the slice except for a small band of tissue close to the cut were invaded (Fig. 5A, D). Activity propagated from the stimulation site laterally along the intact and then along the lesioned part of the slice (Fig. 5B, E). In three of five slices, the velocity of the spread in the lesioned part of the slice was significantly lower in infragranular as compared to supragranular layers (Table 1).

In summary, epileptiform activity can be separately initiated in and spread along infra- and supragranular layers. Thus, intact vertical neuronal connections comprising the whole depth of the cortex are not necessary for the spread of epileptiform activity. Horizontal connections in either supra- or infragranular layers preserved by horizontal lesions are sufficient for propagation.

\section{Vertical lesions through supragranular and infragranular layers}

In 19 slices two cuts separated by $900-2000 \mu \mathrm{m}$ were made: a lateral cut through supragranular layers (layers I, II and III, occasionally including layer IV), and a medial cut through infragranular layers (layers V, VI and the white matter, occasionally including layer IV). The results did not depend on the inclusion or exclusion of layer IV from the cut. In all slices, stimulation electrodes were placed between the two cuts. In this way, neuronal connections only in supragranular layers were available for the spread of epileptiform activity to the medial edge of the slice, and only in infragranular layers for the spread of activity to the lateral edge of the slice.

In all slices, epileptiform activity spread past the infragranular lesion using supragranular neuronal connections only (Figs 6-9). Amplitude declined locally around the lesion, but large amplitudes were regained beyond the lesion. Thus, supragranular layers were sufficient for the spread of epileptiform activity. On the other hand, spread past the supragranular lesion using infragranular neuronal connections was impaired. In eight slices, no activity spread past the lesion with both stimulation of white matter and layer I (Fig. 6). In one slice, the spread occurred with stimulation of white matter, but not of layer I. In the remaining ten slices, epileptiform activity spread past the supragranular lesion (Figs 7A, 8B and 9), but with large shifts of onset latency (Figs 7B, 8C and 9). In contrast, shifts of onset latency were small or absent if infragranular layers were lesioned (Figs 8C and 9).

Shifts of onset latency near the cuts were measured in layers III and $\mathrm{V}$ with stimulation of layer I and the white matter (Fig. 10). Shifts were determined by calculating lines of regression through plots of onset latency against distance. Separate lines of regression were determined from the respective stimulation site to the lesion on the one hand and from the lesion to the edge of the slice on the other hand. The shifts of onset latency were the differences of latencies calculated from the regression lines at the intersection with the lesion (Fig. 10). They were significantly larger at the supragranular compared to the infragranular lesion $(P<0.0001$, paired $t$-test $)$.

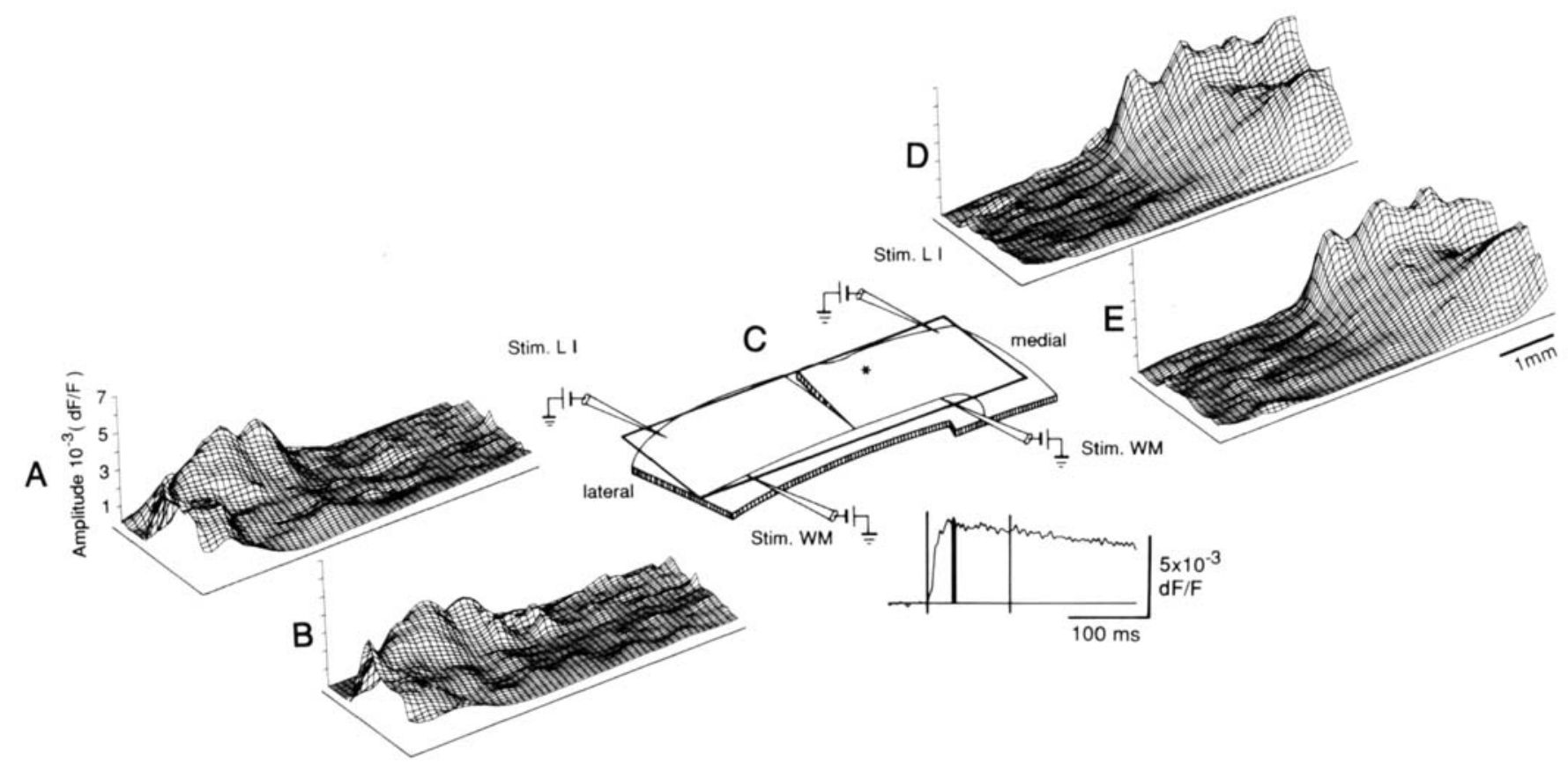

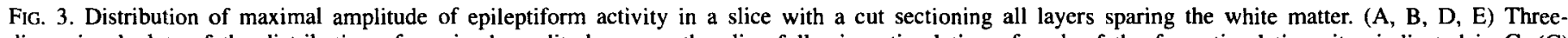

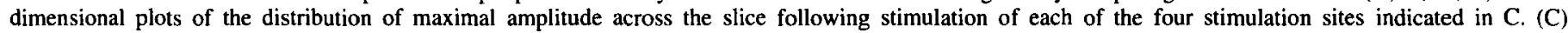

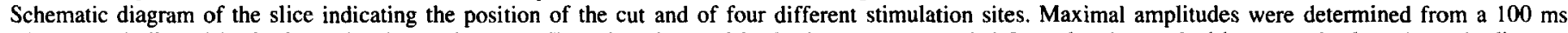

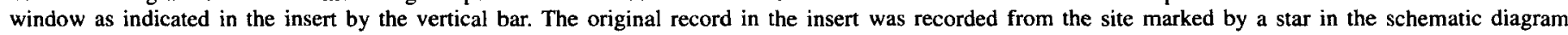

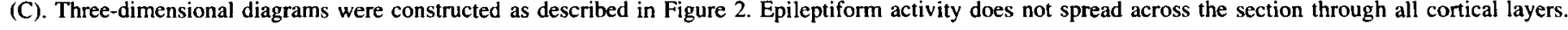


A

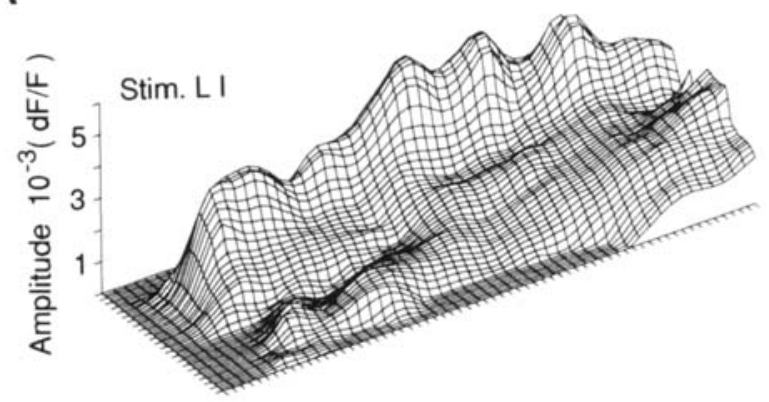

B

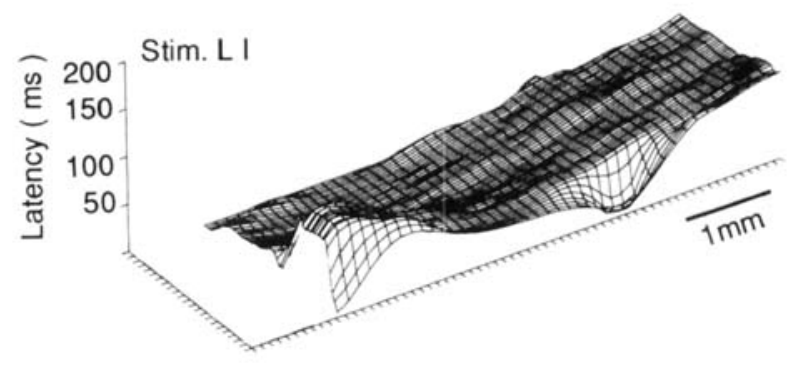

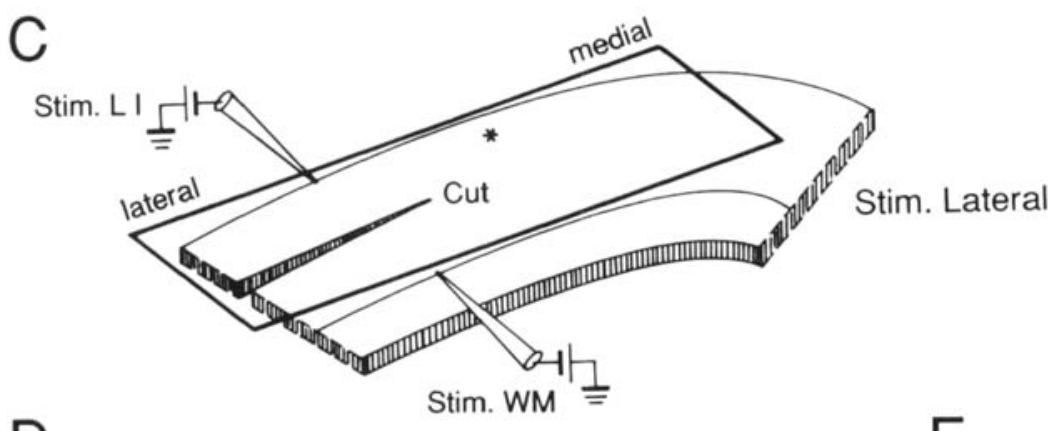

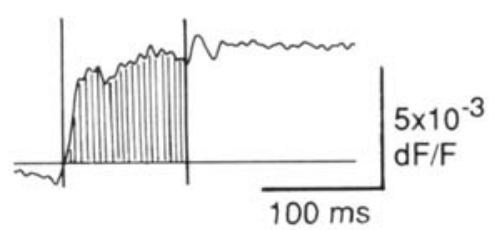

D
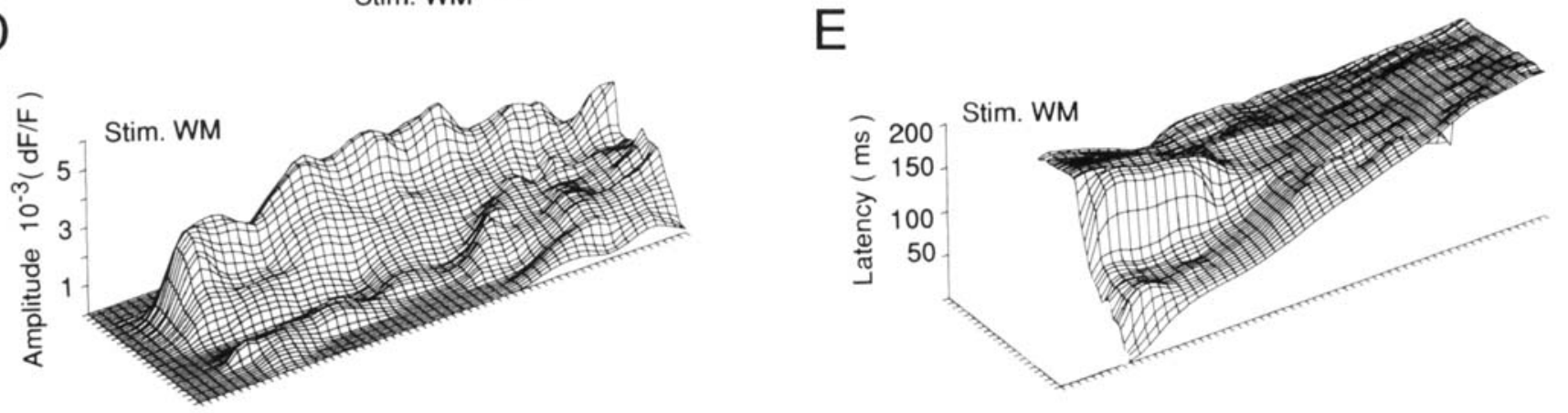

FIG. 4. Distribution of mean window amplitude and onset latency across a slice with a horizontal cut separating supra- and infragranular layers. Stimulation electrodes were placed within the lesioned part of the slice. (A, B, D, E) Three-dimensional plots of the distribution of mean window amplitude (A, D) and onset latency (B, E) across the slice following stimulation of layer I (A, B) and white matter (D, E). (C) Schematic diagram of the slice indicating the position of the cut and of the stimulation electrodes in white matter and layer I. The insert indicates the window ( $100 \mathrm{~ms}$ ) from which mean window amplitude (average of amplitudes in the shaded area) and onset latency were determined. The original record in the insert was recorded from the site marked by a star in the schematic diagram (C). Three-dimensional diagrams were constructed as described in Figure 2. Epileptiform activity is present across the entire slice with both stimulation sites. Spread starts from the stimulation electrode and continues along the lesioned and intact part of the slice.

The course of propagation and the cause of large shifts of onset latencies are well demonstrated by Figure 7B. Epileptiform activity spread from the stimulation electrode laterally towards the lesion through supragranular layers; then, to circumvent the lesion, only infragranular layers were used for the spread of activity. Past the lesion, supragranular layers were invaded again. Frequently, activity re-entered supragranular layers not immediately beyond the lesion, but at a distance of several hundred micrometres. From here, activity propagated both medially and laterally, i.e. both back towards the lesion and to the edge of the slice. As a consequence, potentials approached the lesion from both sides, such that two potentials at different latencies appear near the cut (Fig. 9B, arrows). In these slices, activity propagated into opposite directions and frequently at different velocities along infra- and supragranular layers for $\sim 1 \mathrm{~mm}$ beyond the lesion. At the infragranular lesion, activity propagated uninterrupted in all slices along supragranular layers.

In four slices, additional stimulation sites were chosen at the far medial and/or far lateral side of the slice. In these cases, activity had to approach one lesion from a different direction. This is of importance, since the pattern of spread can depend on direction (Chervin et al., 1988). Also, both lesions had to be passed consecutively. If stimulation electrodes were placed medially, activity had to pass the infragranular lesion prior to the supragranular lesion. In this case, activity spread past the infragranular, but not past the supragranular lesion. If stimulation electrodes were placed laterally, activity spread across both the supragranular and then the infragranular lesion; however, shifts of onset latency were observed at both impediments.

These data indicate the predominant role of horizontal connections 
A

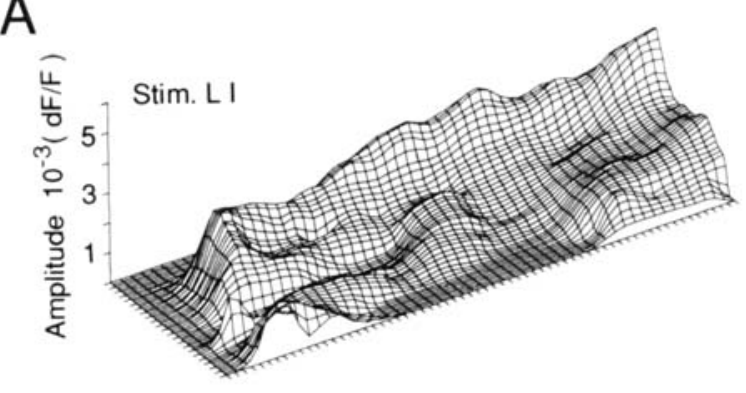

B

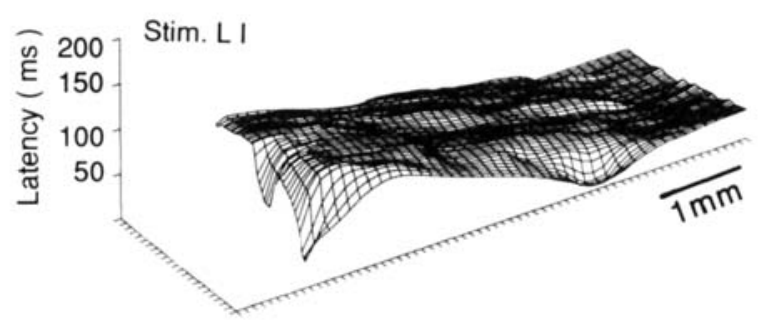

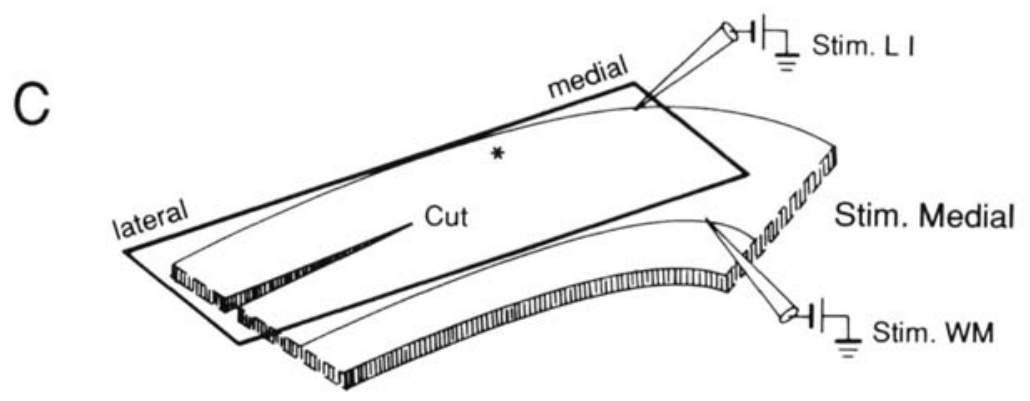

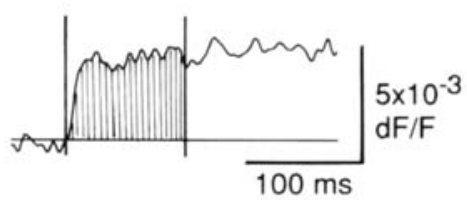

D

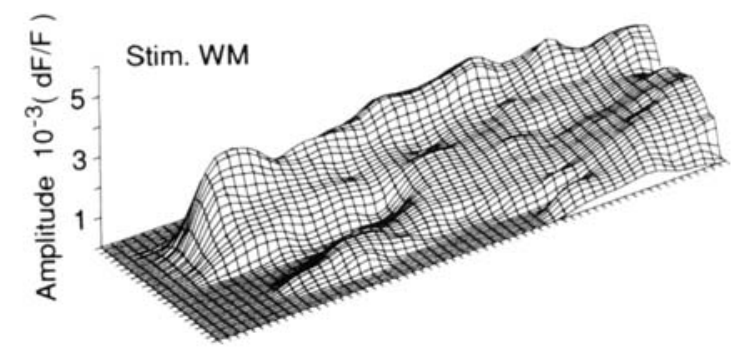

$\mathrm{E}$

FIG. 5. Distribution of mean window amplitude and onset latency across a slice with a horizontal cut separating supra- and infragranular layers. Stimulation electrodes were placed within the intact part of the slice. (A, B, D, E) Three-dimensional plots of the distribution of mean window amplitude (A, D) and onset latency (B, E) across the slice following stimulation of layer I (A, B) and white matter (D, E). (C) Schematic diagram of the slice indicating the position of the cut and of the stimulation electrodes in white matter and layer $\mathbf{I}$. The insert indicates the window ( $100 \mathrm{~ms})$ from which mean window amplitude (average of amplitudes in the shaded area) and onset latency were determined. The original record in the insert was recorded from the site marked by a star in the schematic diagram (C). Three-dimensional diagrams were constructed as described in Figure 2. Epileptiform activity is present across the entire slice with both stimulation sites. Spread starts from the stimulation electrode and continues along both the intact and the lesioned part of the slice.

TABLE 1. Velocity of the spread of epileptiform activity in infragranular and supragranular layers of the intact and lesioned part of the slice

\begin{tabular}{|c|c|c|c|c|c|c|}
\hline \multirow[t]{2}{*}{ Slice } & \multicolumn{3}{|l|}{ Intact part of slice } & \multicolumn{3}{|l|}{ Lesioned part of slice } \\
\hline & Supragranular layers & Infragranular layers & Signif. & Supragranular layers & Infragranular layers & Signif. \\
\hline 1 & $44.2 \pm 1.4$ & $33.9 \pm 2.4$ & s. & $61.6 \pm 3.4$ & $40.9 \pm 2.6$ & $\mathbf{s}$ \\
\hline 2 & $58.8 \pm 1.7$ & $51.2 \pm 2.5$ & n.s. & $90.5 \pm 19.4$ & $108.3 \pm 32.0$ & n.s. \\
\hline 3 & $59.9 \pm 16.0$ & $74.6 \pm 29.9$ & n.s. & $32.9 \pm 2.6$ & $19.7 \pm 2.2$ & s. \\
\hline 4 & $48.0 \pm 1.9$ & $47.1 \pm 3.1$ & n.s. & $36.5 \pm 1.5$ & $43.0 \pm 17.0$ & n.s. \\
\hline 5 & $59.5 \pm 1.9$ & $52.6 \pm 3.1$ & n.s. & $40.5 \pm 1.6$ & $20.9 \pm 3.8$ & s. \\
\hline
\end{tabular}

In five slices with a horizontal cut and distant stimulation sites, the velocity of the spread of activity was calculated from lines of linear regression through plots of onset latencies against distance. Velocities for supragranular layers were determined along layer III, those for infragranular layers along layer V. The values for both stimulation sites (white matter, layer I), repeated measurements, and two rows of photodiodes were pooled. For each slice and for the intact and lesioned part of that slice the significance of difference between the velocity of the spread in infragranular and supragranular layers was determined by use of Student's paired $t$-test (level of significance $P<0.001$ ). Values are mean values \pm SD in $\mathrm{mm} / \mathrm{s}$. Signif., significance of difference: $\mathbf{s}$., significant; n.s., not significant. 


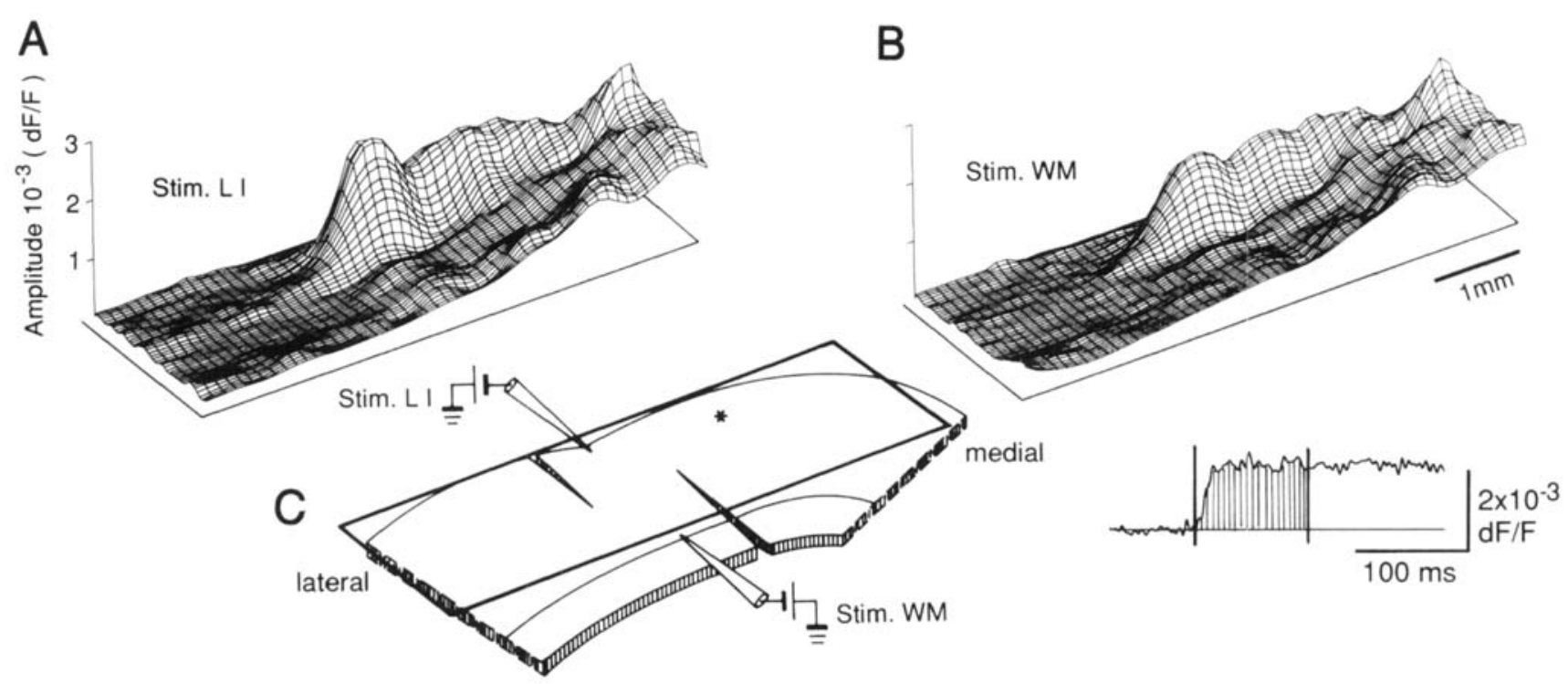

Fig. 6. Distribution of mean window amplitude of epileptiform activity in a slice with two cuts sectioning supragranular layers laterally and infragranular layers medially. (A, B) Three-dimensional plots of the distribution of mean window amplitude across the slice following stimulation of layer I (A) and white matter (B). (C) Schematic diagram of the slice indicating the positions of the cuts and of the stimulation sites. Mean window amplitude (average of amplitudes in the shaded area) was determined from a $100 \mathrm{~ms}$ window as indicated in the insert. The original record in the insert was recorded from the site marked by a star in the schematic diagram $(\mathrm{C})$. Three-dimensional diagrams were constructed as described in Figure 2 . In this example, epileptiform activity spread only medially past the lesion through infragranular layers, but not laterally past the lesion through supragranular layers.

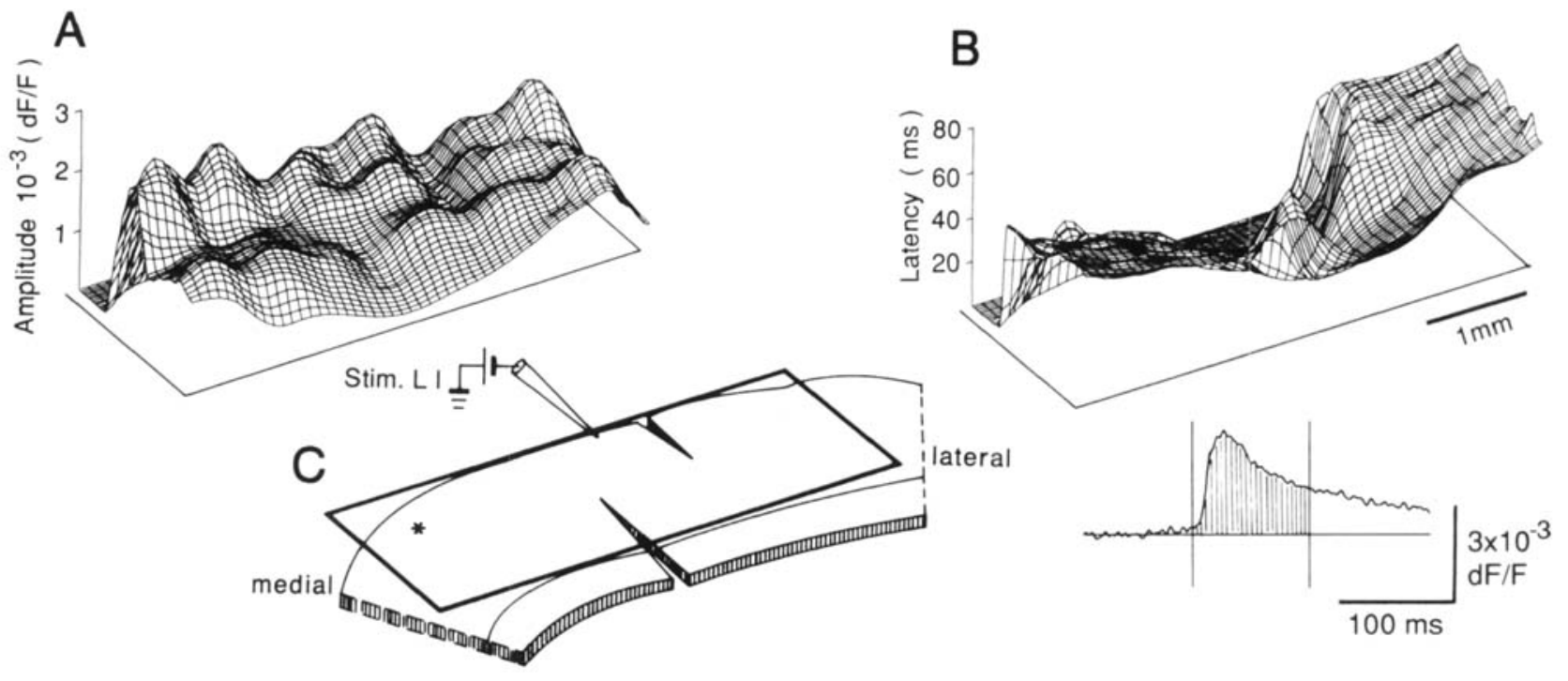

Fig. 7. Distribution of mean window amplitude and onset latency of epileptiform activity in a slice with two cuts sectioning supragranular layers laterally and infragranular layers medially. (A, B) Three-dimensional plots of the distribution of mean window amplitude (A) and onset latency (B) across the slice following stimulation of layer I. (C) Schematic diagram of the slice indicating the positions of the cuts and of the stimulation site. Mean window area (average of amplitudes in the shaded area) and onset latency were determined from a $100 \mathrm{~ms}$ window as indicated in the insert. The original record in the insert was recorded from the site marked by a star in the schematic diagram (C). Three-dimensional diagrams were constructed as described in Figure 2 . In this example, epileptiform activity spread across the entire slice past both lesions through infra- and supragranular layers.

in supragranular cortical layers for the propagation of epileptiform activity.

\section{Discussion}

In the present investigation, epileptiform activity was monitored across neocortical slices using the voltage-sensitive indicator RH795.
As described previously (Albowitz and Kuhnt, 1993c), the distribution of amplitude was highly variable among slices and at different positions within one slice, but the largest amplitudes were always found in layers II and III.

Specific lesions were placed in order to identify neuronal structures necessary and sufficient for the initiation and spread of paroxysmal activity evoked by electrical stimulation and pharmacolo- 


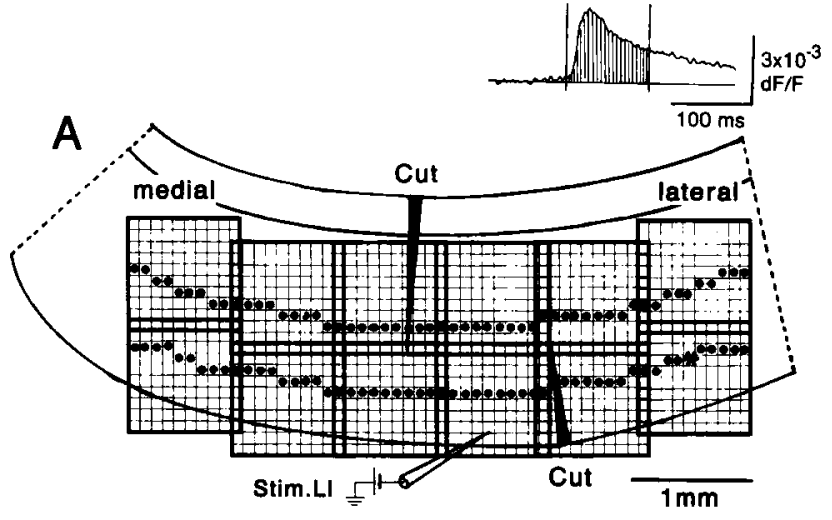

B
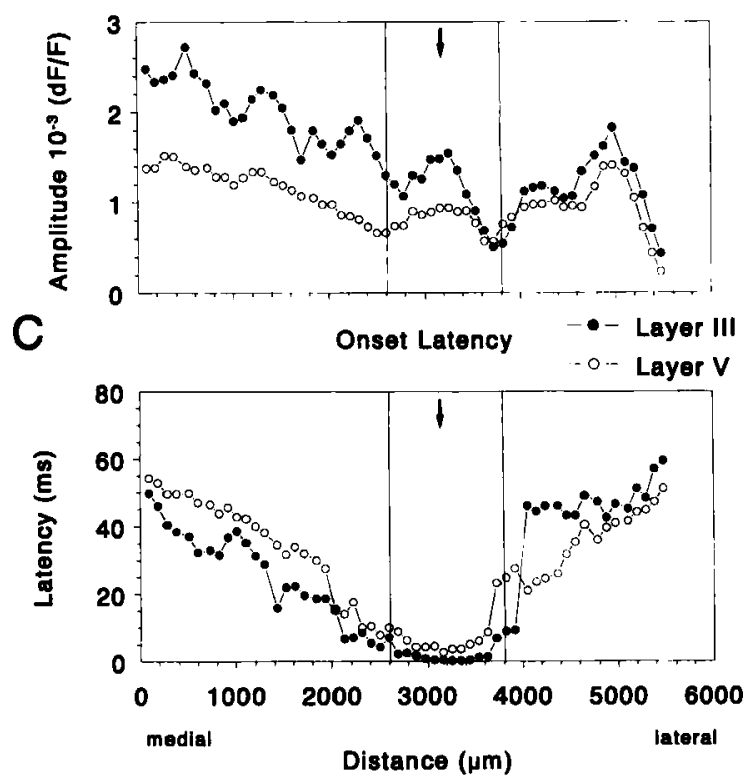

FIG. 8. Values of mean window amplitude and onset latency along layers III and $\mathrm{V}$ in a slice with two cuts sectioning infragranular layers medially and supragranular layers laterally. Same experiment as in Figure 7. (A) Schematic drawing of the slice, indicating the position of the cuts, of the photodiode array and of the stimulation electrode in layer I. Two rows of photodiodes representing layers III and V are marked. The insert on top illustrates the setting of the window $(100 \mathrm{~ms})$ from which mean window amplitude (average of amplitudes in the shaded area) and onset latency were determined. The original record in the insert was recorded from the site marked by a star in the schematic diagram (A). (B, C) Values of mean window amplitude (B) and onset latency $(\mathrm{C})$ from records of photodiodes marked in $\mathrm{A}$ plotted from medial to lateral following stimulation of layer I along layer III (filled symbols) and V (open symbols). Vertical lines indicate the location of the cuts, the arrows the position of the stimulation electrode. Note the shift in latency at the supragranular lesion in C.

gical disinhibition. This methodological approach is based on the assumption that mechanisms other than synaptic transmission, such as 'ephaptic' interactions (Taylor and Dudek, 1984a, b) are not significantly involved in the propagation of epileptiform activity. Ephaptic interactions might persist if the tissue is mechanically cut but the borders are still touching each other. However, cuts through the entire depth of the grey matter interrupted the propagation of epileptiform events in all cases. Therefore, under the present experimental conditions non-synaptic mechanisms, if present, are not strong enough to assure propagation of epileptiform activity across a lesion.

The experiments showed that intracortical connections are necessary and sufficient for the propagation of epileptiform activity in the slice. Further, the separation of supra- and infragranular layers by a horizontal cut could not hinder the initiation and propagation of epileptiform activity in both of the isolated parts. Thus, the intact neuronal structures and connections in either supra- or infragranular layers are sufficient to propagate epileptiform activity following initiation after stimulation of layer $I$ and the white matter respectively. However, neuronal structures in supragranular layers and their horizontal connections are more prone to the propagation of epileptiform activity than those of the infragranular layers. Disruption of horizontal connections in supragranular layers blocked the propagation altogether in some slices and slowed it down in others. Disruption of infragranular horizontal connections never had similar consequences. The variability of changes after supragranular lesions might be caused by different features, such as the magnitude and duration of single-cell depolarization, the degree of synchronization, and other unknown changes due to preparation and storage.

The present results are in contrast to conclusions by Connors and his group (Connors, 1984; Chagnac-Amitai and Connors, 1989b). Based on extensive intracellular recordings, they ascribed a pivotal role in the initiation, synchronization, and the spread of epileptiform discharge to intrinsically bursting neurons of layer V. Here, supragranular layers were not only sufficient but were also preferred for the initiation and propagation of epileptiform activity. This does not exclude the possibility that intrinsically bursting neurons of layer $\mathrm{V}$ play a role if connections are intact, but they are not a necessary requirement.

Due to methodological restrictions (thickness of slice, spatial and temporal resolution, sensitivity of the indicator) no particular importance in the initiation and the spread could be assigned to a specific subsystem in supragranular layers. Several systems of horizontally oriented connections in supragranular layers are possible. Long-range fibres in layer I provide a strong synaptic input to apical dendrites of pyramidal cells (Cauller and Connors, 1994). These longrange axons originate from neurons in neighbouring cortical areas (Rockland and Vigra, 1989). However, in the slice their involvement in the spread of epileptiform activity is unlikely. Firstly, cell bodies giving source to these fibres might be missing in the slice preparation. Secondly, even if these neurons survive, their axons would pass the white matter before entering layer I (Rockland and Vigra, 1989). As was shown by vertical lesions through the entire grey matter, white matter pathways alone are not sufficient to propagate epileptic activity.

Besides this cortico-cortical system, all cortical layers contain short- and long-range horizontal excitatory connections, even though the latter are relatively sparse (Fisken $e t$ al., 1975; Creutzfeldt $e t$ al., 1977). These connections are ideal candidates for the propagation of epileptiform activity. The impact of some of the long-range fibres might be of great functional importance because of their organization in patches in many species (e.g. Kisvárday and Eysel, 1992). These fibres originate from pyramidal cells in layer III and V, and course horizontally for several millimetres before terminating in the same layer by forming dense axonal arborizations which contact groups of postsynaptic neurons (Gilbert and Wiesel, 1983; Kisvárday et al., 1986). Based on the non-uniform propagation of epileptiform activity, their participation in the spread of activity has been suggested (Chervin et al., 1988; Wadman and Gutnick, 1993). Our finding that activity circumventing a supragranular lesion along infragranular pathways initiated epileptiform activity in supragranular layers not 


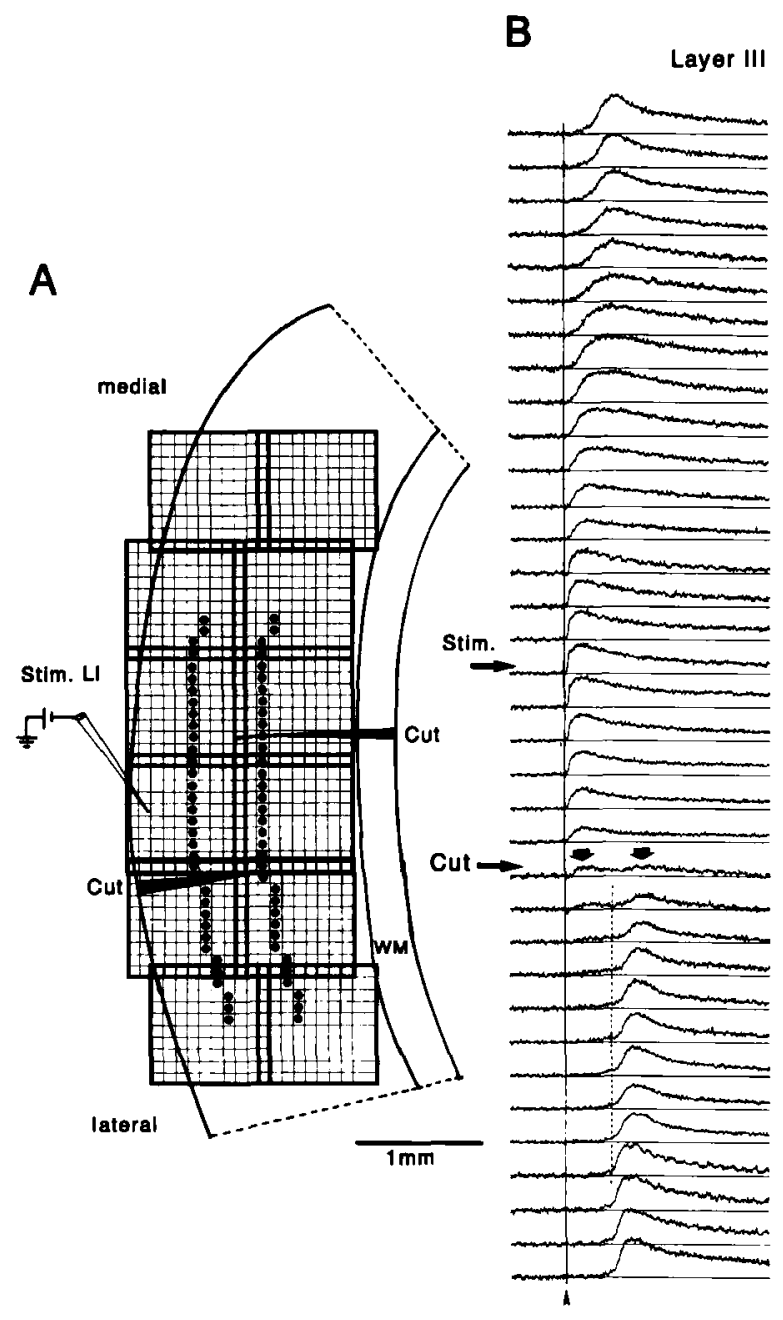

C

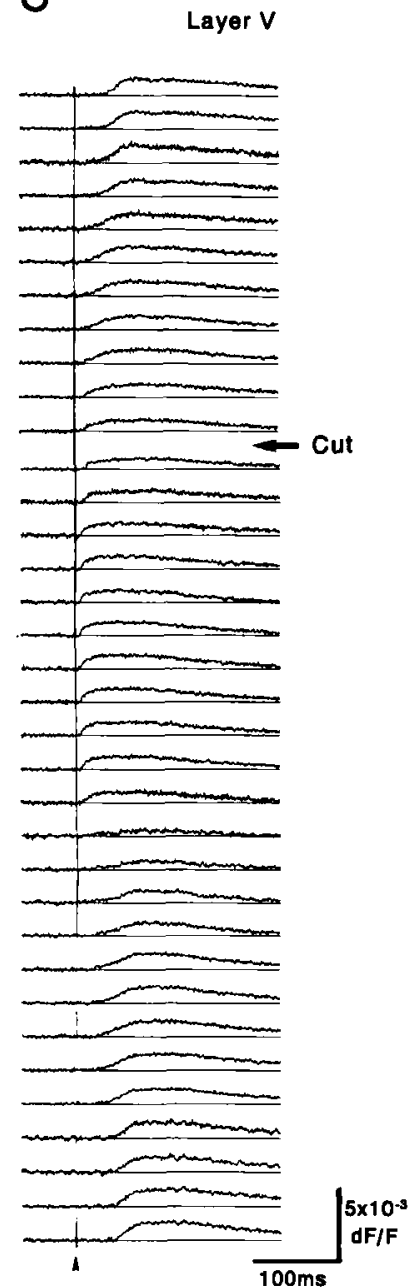

FIG. 9. Photodiode records from layers III and V in a slice with two cuts sectioning infragranular layers medially and supragranular layers laterally. Same experiment as in Figures 7 and 8. (A) Schematic drawing of the slice, indicating the position of the cuts, of the photodiode array and of the stimulation electrode in layer I. Two rows of photodiodes representing parts of layers III and V are marked. (B, C) Photodiode records from the diodes marked in A shown from medial to lateral for layer III (B) and layer V (C). The positions of the stimulation electrode and of the lesions are marked by large arrows, the time points of stimulation are marked by small arrows and by vertical lines. The stippled line marks the shortest onset latency of potentials lateral to the lesion in layer III and indicates the direction of spread from lateral towards the lesion. Note the presence of two separate potentials at the lesion (arrows).

immediately past the lesion, but frequently several hundred micrometres further away, is in favour of this suggestion. The patch system alone is probably not sufficient to propagate epileptiform activity; the velocity of spread measured in these experiments and by others (Chervin et al., 1988; Chagnac-Amitai and Connors, 1989a; Wadman and Gutnick, 1993) is too slow. It could, however, 'predepolarize' the neurons concerned. Activity conducted by short-range fibres would be superimposed to initiate then paroxysmal discharges.

The horizontal connectivity of supra- versus infragranular layers alone is probably not sufficient to explain the observed dominance of supragranular layers for the spread of activity, even if the quantitatively uneven distribution of horizontal connections in different layers is considered (e.g. Burkhalter, 1989). Additional physiological and morphological factors which might facilitate the spread of activity in supragranular layers have to be considered.

Morphological features such as dendritic bundles (Fleischauer et al., 1972), which reach their best expression in layer III, might be an important factor in the synchronization and amplification of activity. Effective synchronization within bundles might be mediated by ephaptic field effects and/or dendro-dendritic synapses (gap junctions; Sloper, 1972). Accordingly, layer II/III has the highest labelling for mRNA coding for specific gap junction proteins (Micevych and Abelson, 1991). The distribution of $N$-methyl-Daspartate (NMDA) receptors, also reaching highest densities in supragranular layers (Rosier et al.. 1993), could be another factor favouring the initiation and the spread in these layers.

The preference for supragranular connections is in accordance with investigations of normal activity in the neocortical slice, which point to strong excitatory interactions in layer III (Langdon and Sur, 1990) and particularly among pyramidal neurons of this layer (Thomson and West, 1993). These excitatory connections are stronger in layer III compared to layer V (Nicoll and Blakemore, 1993). Largest depolarizations evoked by stimulation of either the white matter or layer I were also found in layer II/III using optical techniques (Albowitz and Kuhnt, 1993a). Besides the unequal excitatory efficiency in different layers, there is also evidence that inhibition is more 


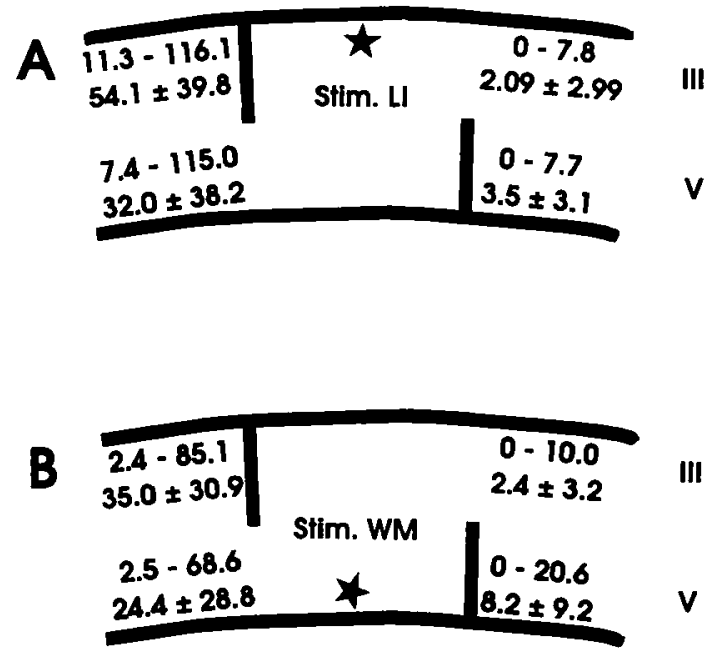

Fig. 10. Shifts of onset latency in layers III and V following stimulation of layer I (A) and the white matter (B) at the supragranular and infragranular lesion. The values provide the range, mean and standard deviation of onset latency shifts in ms, based on $n=10$ for supragranular and $n=18$ for infragranular lesions.

marked in upper layers (Langenstroth et al., 1993). Finally, the spread of normal activity in the slice evoked by stimulation of white matter or layer I is wider in layer III compared to other layers (Luhmann et al., 1990; Albowitz and Kuhnt, 1993c). During epileptiform activity, the particularly large depolarization of layer II/III as compared to other layers is even more pronounced (Albowitz et al., 1990). Thus, the predominant role of supragranular layers in the spread of activity might be based on the higher depolarization, which (in the present model of epilepsy) is further increased and broadened by blockage of inhibitory activity. The particularly large and broad depolarization might open more NMDA receptor-activated channels as well as voltage-dependent calcium channels. As a consequence, horizontal connections in supragranular layers are activated more strongly and (at least in part) earlier than in infragranular layers, leading to more efficient temporal and spatial summation of depolarization at their synaptic targets, and finally to the generation of epileptiform discharge at the distant site.

It is, however, also evident that for the expression of epileptiform activity the connectivity used during normal stimulation must be supplemented by other excitatory ones (or be devoid of various inhibitory interactions) or must be activated in a different temporal pattern. This conclusion is derived from the fact that full-blown epileptiform discharges are generally not observed along the stimulation axis but only at a certain distance (unpublished observation).

In summary, horizontal connections in supragranular layers are of particular importance for the propagation of epileptiform activity in the slice preparation. Horizontal pathways in infracortical layers and vertical connections across all layers might contribute to the spread and facilitate synchronization, but they are not essential.

\section{Acknowledgements}

We are grateful to Mrs S. Lausmann and Mrs U. Steveling for careful histological preparations, and to Mrs A. Tlustochowski for skilful technical assistance. This work was supported by Deutsche Forschungsgemeinschaft grants Al 268/3-1 and $\mathrm{Al} 268 / 3-2$ to B. A.

\section{References}

Albowitz, B. and Kuhnt, U. (1993a) Evoked changes of membrane potential in guinea pig sensory neocortical slices, an analysis with voltage sensitive dyes and a fast optical recording method. Exp. Brain Res., 93, 213-225.

Albowitz, B. and Kuhnt, U. (1993b) Spread of epileptiform potentials in the neocortical slice: recordings with voltage-sensitive dyes. Brain Res., 631, $329-333$.

Albowitz, B, and Kuhnt, U. (1993c) The contribution of intracortical connections to horizontal spread of activity in the neocortex as revealed by voltage sensitive dyes and a fast optical recording method. Eur. J. Neurosci., 5, 1349-1359

Albowitz, B. and Kuhnt, U. (1993d) Optical recordings reveal that epileptiform activity in neocortical slices spreads preferentially through upper cortical layers. Soc. Neurosci. Abstr, 19, 1032.

Albowitz, B., Kuhnt, U. and Ehrenreich, L. (1990) Optical recording of epileptiform voltage changes in the neocortical slice. Exp. Brain Res., 81, 241-256.

Braitenberg, V. and Schuz, A. (1991) Anatomy of the Cortex. Statistics and Geometry. Springer-Verlag, Berlin.

Burkhalter, A. (1989) Intrinsic connections of rat primary visual cortex, laminar organization of axonal projections. J. Comp. Neurol., 179, 171-186.

Cauller, L. J. and Connors, B. W. (1994) Synaptic physiology of horizontal afferents to layer I in slices of rat SI neocortex. J. Neurosci., 14, 751-762.

Chagnac-Amitai, Y. and Connors, B. W. (1989a) Horizontal spread of synchronized activity in neocortex and its control by GABA-mediated inhibition. J. Neurophysiol., 61, 747-758.

Chagnac-Amitai, Y. and Connors, B. W. (1989b) Synchronized excitation and inhibition driven by intrinsically bursting neurons in neocortex. J. Neurophysiol., 62, 1149-1162.

Chervin, R. D., Pierce, P. A. and Connors, B. W. (1988) Periodicity and directionality in the propagation of epileptiform discharges across neocortex. J. Neurophysiol., 60, 1695-1713.

Connors, B. W. (1984) Initiation of synchronized bursting in neocortex. Nature, 310, 685-687.

Creutzfeldt, O. D., Garey, L. J., Kuroda, R. and Wolff, J. R. (1977) The distribution of degenerating axons after small lesions in the intact and isolated visual cortex of the cat. Exp. Brain Res., 27, 419-440.

Fisken, R. S., Garey, L. J. and Powell, T. P. S. (1975) The intrinsic, association and commissural connections of area 17 in the visual cortex. Phil. Trans. $R$. Soc. Lond. Ser. B, 272, 487-536.

Fleischauer, K., Petsche, H. and Wittkowski, W. (1972) Vertical bundles of dendrites in the neocortex. Z. Anat. Entwicklungsgesch., 136, 213-223.

Gilbert, C. D. and Kelly, J. P. (1975) The projection of cells in different layers of the cat's visual cortex. J. Comp. Neurol., 163, 81-106.

Gilbert, C. D. and Wiesel, T. N. (1979) Morphology and intracortical projections of functionally characterised neurons in the cat visual cortex. Nature, 280, 120-125.

Gilbert, C. D. and Wiesel, T. N. (1983) Clustered intrinsic connections in cat visual cortex. J. Neurosci., 3, 1116-1133.

Goldensohn, E. S. and Salazar, A. M. (1986) Temporal and spatial distribution of intracellular potentials during generation and the spread of epileptogenic discharge. Adv. Neurol., 44, 559-582.

Grinvald, A., Lieke, E., Frostig, R. D., Gilbert, C. D. and Wiesel, T. N. (1986) Functional architecture of cortex revealed by optical imaging of intrinsic signals. Nature, 324, 361-364.

Gutnick, M. J. and Prince, D. A. (1975) Effects of projected cortical epileptiform discharges on neuronal activities in ventrobasal thalamus of the cat: ictal discharges. Exp. Neurol., 46, 418-491.

Gutnick, M. J., Connors, B. W. and Prince, D. A. (1982) Mechanisms of neocortical epileptogenesis in vitro. J. Neurophysiol., 48, 1321-1162.

Kisvárday, Z. F. and Eysel, U. T. (1992) Cellular organization of reciprocal patchy networks in layer III of cat visual cortex (area 17). Neuroscience, 46, 275-286.

Kisvárday, Z. F., Martin, K. A. C., Freund, T. F., Maglóczky, Zs., Whitteridge, D. and Somogyi, P. (1986) Synaptic targets of HRP-filled layer III pyramidal cells in the cat striate cortex. Exp. Brain Res., 64, 541-552.

Kusske, J. A. (1976) Interaction between thalamus and cortex in experimental epilepsy in the cat. Exp. Neurol, 50, 568-578.

Langdon, R. B. and Sur, M. (1990) Components of field potentials evoked by white matter stimulation in isolated slices of primary visual cortex, spatial distribution and synaptic order. $J$. Neurophysiol., 64, 1484-1501.

Langenstroth, M., Albowitz, B. and Kuhnt, U. (1993) Spatio-temporal distribution of GABA-mediated inhibitory effects in neocortical slices of 
the guinea pig. In Elnser, N. and Heisenberg, M. (eds), Gene-BrainBehavior. Proceedings of the 2lst Göttingen Neurobiology Conference. Thieme, Stuttgart, p. 494.

Luhmann, H. J., Greuel, J. M. and Singer, W. (1990) Horizontal interactions in cat striate cortex, II. A current source-density analysis. Eur. J. Neurosci. 2, 358-368.

London, J. A., Cohen, L. B. and Wu, J.-Y. (1989) Optical recordings of cortical response to whisker stimulation before and after the addition of an epileptogenic agent. J. Neurosci., 9, 2182-2190.

Martin, K. A. C. and Whitteridge, D. (1984) Form, function and intracortical projections of spiny neurons in the striate cortex of the cat. J. Physiol. (Lond.), 353, 463-504.

Matsumoto, H. and Ajmone-Marsan, C. (1964) Cortical cellular phenomena in experimental epilepsy, ictal manifestations. Exp. Neurol., 9, 305-326.

Micevych, P. E. and Abelson, L. (1991) Distribution of mRNA coding for liver and heart gap junctions proteins in the rat central nervous system. J. Comp. Neurol., 305, 96-118.

Nicoll, A. and Blakemore, C. (1993) Patterns of local connectivity in the neocortex. Neural Computat., 5, 665-680.

Rockland, K. S. and Vigra, A. (1989) Terminal arbors of individual 'feedback' axons projecting from $\mathrm{V} 2$ to $\mathrm{V} 1$ in the macaque monkey: a study using immunohistochemistry of anterogradely transported Phaseolus vulgaris leucoagglutinin. J. Comp. Neurol., 285, 54-72.

Rosier, A. M., Arckens, L., Orban, G. A. and Vandesande, F. (1993) Laminar distribution of NMDA receptors in cat and monkey visual cortex visualized by $\left({ }^{3} \mathrm{H}\right)-\mathrm{MK}-801$ binding. J. Comp. Neurol., 335, 369-380.
Schwartzkroin, P. A., Mutani, R. and Prince, D. A. (1975) Orthodromic and antidromic effects of a cortical epileptiform focus on ventrolateral nucleus in the cat. J. Neurophysiol., 38, 795-811.

Sloper, J. J. (1972) Gap junctions between dendrites in the primate neocortex Brain Res., 44, 641-646.

Taylor, C. P. and Dudek, F. E. (1984a) Excitation of hippocampal pyramidal cells by an electrical field effect. $J$. Neurophysiol., 52, 126-142.

Taylor, C. P. and Dudek, F. E. (1984b) Synchronization without active chemical synapses during hippocampal afterdischarges. J. Neurophysiol., 52, 143-155.

Tigges, J. and Tigges, M. (1982) Principles of axonal collateralization of laminae II-III pyramids in area 17 of squirrel monkey: a quantitative Golgi study. Neurosci. Lett., 29, 99-104.

Thomson, A. M. and West, D. C. (1993) Fluctuations in pyramid-pyramid excitatory postsynaptic potentials modified by presynaptic firing pattern and postsynaptic membrane potential using paired intracellular recordings in rat neocortex. Neuroscience, 54, 329-346.

Traub, R. D. and Wong, R. K. S. (1982) Cellutar mechanisms of neuronal synchronization in epilepsy. Science, 216, 745-747.

Wadman, W. J. and Gutnick, M. J. (1993) Non-uniform propagation of epileptiform discharge in brain slices of rat neocortex. Neuroscience, $\mathbf{5 2}$, 255-262.

Wong, B. Y. and Prince, D. A. (1990) The lateral spread of ictal discharge in neocortical brain slices. Epilepsy Res., 7, 29-39.

Wree, A., Zilles, K. and Schleicher, A. (1981) A quantitative approach to cytoarchitecture, VII. The areal pattern of the cortex of the guinea pig. Anat. Embryol, 162, 81-103. 